\title{
Persistence of dengue (serotype 2 and 3), zika, yellow fever, and murine hepatitis virus RNA in untreated wastewater
}

Checklist MIQE Table

\begin{tabular}{|c|c|c|}
\hline Item to check & Importance & Remarks \\
\hline \multicolumn{3}{|l|}{ Experimental design } \\
\hline Definition of experimental and control groups & $\begin{array}{l}\text { E } \\
\text { (Essential) }\end{array}$ & $\begin{array}{l}\text { Control group: Viral RNA } \\
\text { extracted from microcosms at T0 } \\
\text { prior to incubation but after } \\
\text { treatment (i.e. heat inactivation for } \\
\text { one group). } \\
\text { Experimental group: Viral RNA } \\
\text { extracted from microcosms at } \\
\text { subsequent sampling times. }\end{array}$ \\
\hline Number within each group & $\mathrm{E}$ & $\begin{array}{l}\text { Each treatment and temperature } \\
\text { have triplicates }(\mathrm{N}=3) \text {, individual } \\
\text { replicates were assayed with two } \\
\text { RT-qPCR reaction replicate }(\mathrm{n}=2)\end{array}$ \\
\hline $\begin{array}{l}\text { Assay carried out by the core or investigator's } \\
\text { laboratory? }\end{array}$ & $\begin{array}{l}\text { D } \\
\text { (Desired) }\end{array}$ & Investigator's laboratory \\
\hline Acknowledgment of authors' contributions & $\mathrm{D}$ & - \\
\hline \multicolumn{3}{|l|}{ Sample } \\
\hline Description & $\mathrm{E}$ & $\begin{array}{l}\text { Viral RNA of YFV, ZIKV, } \\
\text { DENV-2, DENV-3, and MHV } \\
\text { extracted from microcosm. }\end{array}$ \\
\hline Volume/mass of sample processed & $\mathrm{D}$ & Not applicable \\
\hline Microdissection or macrodissection & $\mathrm{E}$ & Not applicable \\
\hline Processing procedure & $\mathrm{E}$ & $\begin{array}{l}\text { Before sample extraction, each } \\
\text { microcosm was vortexed for } 15 \mathrm{~s} \text { to } \\
\text { ensure homogeneity. A } 140 \mathrm{uL} \\
\text { aliquot was taken and viral RNA } \\
\text { extracted according to Qiagen } \\
\text { QIAmp Viral RNA Kit protocol. } \\
\text { We extracted nuclease-free water } \\
\text { (together with the samples) at each } \\
\text { sampling time as negative control } \\
\text { to monitor cross-contamination. } \\
\text { No cross-contamination was } \\
\text { detected throughout the study. } \\
\text { Extraction efficiency between } \\
\text { sampling days was controlled by } \\
\text { monitoring pepper mild mottle } \\
\text { virus (PMMoV) levels inherently } \\
\text { present within the microcosm. } \\
\text { PMMoV was chosen due to its } \\
\text { persistence over a wide range of } \\
\text { environmental conditions and } \\
\text { temperature. We found that } \\
\text { PMMoV levels for all time points }\end{array}$ \\
\hline
\end{tabular}




\begin{tabular}{|c|c|c|}
\hline & & $\begin{array}{l}\text { and across all treatments to be } \\
\text { highly consistent }(\mathrm{Cq}=36.46 \pm \\
0.46 ; \text { mean } \pm \mathrm{SD}) .\end{array}$ \\
\hline If frozen, how and how quickly? & $\mathrm{E}$ & Placed into $-80^{\circ} \mathrm{C}$ \\
\hline If fixed, with what and how quickly? & $\mathrm{E}$ & Not applicable \\
\hline Sample storage conditions and duration & $\mathrm{E}$ & $\begin{array}{l}\text { Viral RNA was stored in }-80^{\circ} \mathrm{C} \\
\text { and was assayed within one week } \\
\text { of extraction. }\end{array}$ \\
\hline Procedure and/or instrumentation & $\mathrm{E}$ & Not applicable \\
\hline Name of kit and details of any modifications & $\mathrm{E}$ & $\begin{array}{l}\text { QIAmp Viral RNA Kit (Qiagen } \\
\# 52906 \text { ); no modification }\end{array}$ \\
\hline Source of additional reagents used & $\mathrm{D}$ & - \\
\hline Details of DNase or RNase treatment & $\mathrm{E}$ & - \\
\hline Contamination assessment (DNA or RNA) & $\mathrm{E}$ & - \\
\hline Nucleic acid quantification & $\mathrm{E}$ & - \\
\hline Instrument and method & E & - \\
\hline Purity (A260/A280) & $\mathrm{D}$ & - \\
\hline Yield & $\mathrm{D}$ & - \\
\hline RNA integrity: method/instrument & $\mathrm{E}$ & - \\
\hline RIN/RQI or Cq of 3' and 5' transcripts & $\mathrm{E}$ & - \\
\hline Electrophoresis traces & $\mathrm{D}$ & - \\
\hline Inhibition testing (Cq dilutions, spike, or other) & $\mathrm{E}$ & $\begin{array}{l}\text { We defined qPCR inhibition to be } \\
\text { present if there was more than } 60 \% \\
\text { decrease in the RNA quantity of } \\
\text { the undiluted RNA samples when } \\
\text { compared with the } 1: 5 \text { diluted } \\
\text { RNA samples, after adjusting for } \\
\text { the dilution factor by } \\
\text { multiplication of five. We tested } \\
\text { D0 and D3 samples across all } \\
\text { treatments and have determined } \\
\text { that there is no significant } \\
\text { inhibition for all targets when } \\
\text { compared with undiluted samples. }\end{array}$ \\
\hline \multicolumn{3}{|l|}{ Reverse transcription } \\
\hline Complete reaction conditions & $\mathrm{E}$ & $\begin{array}{l}\text { One-step RT-qPCR reactions were } \\
\text { performed. }\end{array}$ \\
\hline Amount of RNA and reaction volume & $\mathrm{E}$ & $\begin{array}{l}1 \text { microlitre of RNA in a final } \\
\text { reaction volume of } 10 \text { microliters. }\end{array}$ \\
\hline $\begin{array}{l}\text { Priming oligonucleotide (if using GSP) and } \\
\text { concentration }\end{array}$ & $\mathrm{E}$ & Not applicable \\
\hline Reverse transcriptase and concentration & $\mathrm{E}$ & $\begin{array}{lr}\text { Taqman Virus } & \text { 1-Step master mix } \\
\text { (Thermofisher } & \# 4444434) \\
\text { following } & \text { manufacturer's } \\
\text { recommendations } & \end{array}$ \\
\hline Temperature and time & $\mathrm{E}$ & $5 \mathrm{~min}$ at $50^{\circ} \mathrm{C}$ \\
\hline Manufacturer of reagents and catalogue numbers & $\mathrm{D}$ & Not applicable \\
\hline Cqs with and without reverse transcription & $\mathrm{D}$ & Not applicable \\
\hline Storage conditions of cDNA & $\mathrm{D}$ & All RNAs are frozen at $-80^{\circ} \mathrm{C}$ \\
\hline
\end{tabular}




\begin{tabular}{|c|c|c|}
\hline qPCR protocol & & \\
\hline Complete reaction conditions & $\mathrm{E}$ & $\begin{array}{l}\text { RT-qPCR was performed using the } \\
\text { Taqman Virus } 1 \text {-Step master mix } \\
\text { (Thermofisher \#4444434) in } \\
\text { duplicates, at a final volume of } 10 \\
\mu \mathrm{L} \text {, according to the } \\
\text { manufacturer's recommendations. } \\
\text { We used } 1 \mu \mathrm{L} \text { of extracted RNA } \\
\text { sample per RT-qPCR sample. A } \\
\text { single reverse or forward primer } \\
\text { and probe was used for each target } \\
\text { and sample. The reactions are } \\
\text { performed on a Bio-Rad CFX } 384 \\
\text { real-time PCR instrument under } \\
\text { the following conditions, } 5 \text { min at } \\
50{ }^{\circ} \mathrm{C} \text { and } 20 \mathrm{~s} \text { at } 95^{\circ} \mathrm{C}, \text { followed } \\
\text { by } 45 \text { cycles of } 3 \mathrm{~s} \text { at } 95^{\circ} \mathrm{C} \text { and } \\
30 \mathrm{~s} \text { at } 60^{\circ} \mathrm{C} \text {. }\end{array}$ \\
\hline Reaction volume and amount of cDNA/DNA & $\mathrm{E}$ & $\begin{array}{l}\text { Final reaction volume of } 10 \mu \mathrm{L} \text {, } \\
\text { containing } 1 \mu \mathrm{L} \text { of RNA. }\end{array}$ \\
\hline Primer, (probe), Mg2, and dNTP concentrations & $\mathrm{E}$ & $\begin{array}{l}\text { The final concentration of the RT- } \\
\text { qPCR primers and probes were } \\
\text { different according to each target: } \\
\text { YFV and ZIKV primers at } 400 \mathrm{nM} \\
\text { each and probe at } 250 \mathrm{nM} \text {, DENV- } \\
2 \text { and DENV-3 primers at } 500 \mathrm{nM} \\
\text { each and probe at } 180 \mathrm{nM} \text {; MHV } \\
\text { primers at } 300 \mathrm{nM} \text { each and probe } \\
\text { at } 400 \mathrm{nM}\end{array}$ \\
\hline Polymerase identity and concentration & $\mathrm{E}$ & $\begin{array}{lr}\text { Taqman Virus 1-Step master mix } \\
\text { (Thermofisher } & \# 4444434 \text { ) } \\
\text { following } & \text { manufacturer's } \\
\text { recommendations } & \end{array}$ \\
\hline Buffer/kit identity and manufacturer & $E$ & Thermofisher \\
\hline Exact chemical composition of the buffer & $\mathrm{D}$ & - \\
\hline Additives (SYBR Green I, DMSO, and so forth) & $\mathrm{E}$ & None \\
\hline $\begin{array}{l}\text { Manufacturer of plates/tubes and catalog } \\
\text { number }\end{array}$ & $\mathrm{D}$ & Bio-Rad HSP3805 \\
\hline Complete thermocycling parameters & $\mathrm{E}$ & $\begin{array}{l}5 \text { min at } 50^{\circ} \mathrm{C} \text { and } 20 \mathrm{~s} \text { at } 95^{\circ} \mathrm{C} \text {, } \\
\text { followed by } 45 \text { cycles of } 3 \mathrm{~s} \text { at } \\
95^{\circ} \mathrm{C} \text { and } 30 \mathrm{~s} \text { at } 60^{\circ} \mathrm{C} \text {. }\end{array}$ \\
\hline Reaction setup (manual/robotic) & $\mathrm{D}$ & $\begin{array}{l}\text { Eppendorf electronic single } \\
\text { channel pipettes }\end{array}$ \\
\hline Manufacturer of qPCR instrument & $\mathrm{D}$ & Bio-Rad \\
\hline \multicolumn{3}{|l|}{ qPCR validation } \\
\hline Evidence of optimization (from gradients) & $\mathrm{D}$ & - \\
\hline Specificity (gel, sequence, melt, or digest) & $\mathrm{E}$ & - \\
\hline For SYBR Green I, Cq of the NTC & $\mathrm{E}$ & Not applicable \\
\hline
\end{tabular}




\begin{tabular}{|c|c|c|}
\hline Calibration curves with slope and y intercept & $\mathrm{E}$ & $\begin{array}{l}\text { Reported at supplementary } \\
\text { material Figure S3 }\end{array}$ \\
\hline PCR efficiency calculated from slope & $\mathrm{E}$ & $\begin{array}{l}\text { Reported at supplementary } \\
\text { material Table S4 }\end{array}$ \\
\hline CIs for PCR efficiency or SE & $\mathrm{D}$ & - \\
\hline $\mathrm{r}^{2}$ of calibration curve & $\mathrm{E}$ & $\begin{array}{l}\text { Reported at supplementary } \\
\text { material Table S4 }\end{array}$ \\
\hline Linear dynamic range & $\mathrm{E}$ & $\begin{array}{l}\text { YFV: } 3 \text { logs; } \text { ZIKV: } 5 \text { logs; } \\
\text { DENV-2: } 5.77 \text { logs; DENV-3: } \\
5.77 \text { logs; MHV: } 3.60 \text { logs }\end{array}$ \\
\hline Cq variation at LOD & $\mathrm{E}$ & $\begin{array}{l}\text { YFV: } 37.078 \pm 0.486 ; \text { ZIKV: } \\
32.724 \pm 0.505 ; \text { DENV-2: } 36.044 \\
\pm 1.097 ; \text { DENV-3: } 35.623 \pm 1.415 \\
\text { MHV: } 34.817 \pm 0.499\end{array}$ \\
\hline CIs throughout range & $\mathrm{D}$ & - \\
\hline Evidence for LOD & $\mathrm{E}$ & - \\
\hline If multiplex, efficiency and LOD of each assay & $\mathrm{E}$ & No multiplex \\
\hline \multicolumn{3}{|l|}{ Data analysis } \\
\hline qPCR analysis program (source, version) & $\mathrm{E}$ & BioRad CFX Manager (v. 3.1) \\
\hline Method of Cq determination & $\mathrm{E}$ & $\begin{array}{l}\text { Automated calculation by } \mathrm{qPCR} \\
\text { analysis program }\end{array}$ \\
\hline Outlier identification and disposition & $\mathrm{E}$ & No outlier \\
\hline Results for NTCs & $\mathrm{E}$ & $\begin{array}{l}\text { No template controls (NTCs) were } \\
\text { included for each run and each } \\
\text { target, and none of them were } \\
\text { amplified. }\end{array}$ \\
\hline $\begin{array}{l}\text { Justification of number and choice of reference } \\
\text { genes }\end{array}$ & $\mathrm{E}$ & Not applicable \\
\hline Description of normalization method & $\mathrm{E}$ & $\begin{array}{l}\text { Viral RNA concentration for each } \\
\text { sampling day were normalized } \\
\text { against viral RNA concentration at } \\
\text { day } 0\end{array}$ \\
\hline $\begin{array}{l}\text { Number and concordance of biological } \\
\text { replicates }\end{array}$ & $\mathrm{D}$ & Three biological replicates \\
\hline $\begin{array}{l}\text { Number and stage (RT or qPCR) of technical } \\
\text { replicates }\end{array}$ & $\mathrm{E}$ & Two RT-qPCR technical replicates \\
\hline Repeatability (intraassay variation) & $\mathrm{E}$ & $\begin{array}{l}\text { Triplicate of quantified RNA } \\
\text { target (see Supplementary } \\
\text { Methods) were added to each plate } \\
\text { and functions as positive controls } \\
\text { and interplate calibrator. } \\
\text { Moreover, all technical replicates } \\
\text { conducted are within 1 Cq of each } \\
\text { other. }\end{array}$ \\
\hline Reproducibility (interassay variation, CV) & $\mathrm{D}$ & - \\
\hline Power analysis & $\mathrm{D}$ & - \\
\hline Statistical methods for results significance & $\mathrm{E}$ & $\begin{array}{l}\text { Comparisons were made using } \\
\text { ANOVA with Holm-Sidak post- } \\
\text { hoc multiple comparison test }\end{array}$ \\
\hline Software (source, version) & $\mathrm{E}$ & GraphPad Prism (version 8.4.3) \\
\hline Cq or raw data submission with RDML & $\mathrm{D}$ & - \\
\hline
\end{tabular}




\begin{tabular}{|l|l|l|}
\hline & & \\
\hline qPCR target information & & \\
\hline Gene symbol & E & - \\
\hline Sequence accession number & E & - \\
\hline Location of amplicon & D & - \\
\hline Amplicon length & E & Reported in Table S3 \\
\hline In silico specificity screen (BLAST, and so on) & E & $\begin{array}{l}\text { Primers specific against targets } \\
\text { (BLAST against nr/nt database) }\end{array}$ \\
\hline $\begin{array}{l}\text { Pseudogenes, retropseudogenes, or other } \\
\text { homologs? }\end{array}$ & D & Not applicable \\
\hline Sequence alignment & D & Not applicable \\
\hline Secondary structure analysis of amplicon & D & Not applicable \\
\hline $\begin{array}{l}\text { Location of each primer by exon or intron (if } \\
\text { applicable) }\end{array}$ & E & Not applicable \\
\hline What splice variants are targeted? & E & Not applicable \\
\hline & & Reported in Table S3 \\
\hline qPCR oligonucleotides & & - \\
\hline Primer sequences & E & Reported in Table S3 \\
\hline RTPrimerDB identification number & D & $\begin{array}{l}\text { 5' FAM and 3'BHQ1 quencher for } \\
\text { probes }\end{array}$ \\
\hline Probe sequences & D & IDT \\
\hline Location and identity of any modifications & E & $\begin{array}{l}\text { Desalting for primers, HPLC for } \\
\text { probes }\end{array}$ \\
\hline Manufacturer of oligonucleotides & D & D \\
\hline Purification method & & \\
\hline
\end{tabular}

